\title{
A CHARACTERIZATION OF DUAL QUERMASSINTEGRALS AND THE ROOTS OF DUAL STEINER POLYNOMIALS
}

\author{
DAVID ALONSO-GUTIÉRREZ, MARTIN HENK, AND MARÍA A. HERNÁNDEZ \\ CIFRE
}

\begin{abstract}
Let $m \geq 1,\left(r_{0}=0, r_{1}, \ldots, r_{m}\right)$ be a tuple of distinct real numbers and $n \geq 2$. We provide a characterization of those tuples $\left(\omega_{0}, \omega_{1}, \ldots, \omega_{m}\right)$ of real numbers such that there exist $n$-dimensional star bodies $K, L$ with $\widetilde{\mathrm{W}}_{r_{j}}(K, L)=\omega_{j}, j=0, \ldots, m$, where $\widetilde{\mathrm{W}}_{r}(K, L)$ denotes the $r$-th dual (relative) quermassintegral of $K$ and $L$. This may be regarded as an analogue within the dual Brunn-Minkowski theory of Shephard's classification of quermassintegrals of two convex bodies.

It turns out that the characterization of dual quermassintegrals is related to the moment problem, and based on this relation, we also derive new determinantal inequalities among the dual quermassintegrals. Moreover, this characterization will be the key tool in order to investigate structural properties of the set of roots of dual Steiner polynomials of star bodies.
\end{abstract}

\section{INTRODUCTION AND NOTATION}

Let $\mathcal{K}^{n}$ denote the set of convex bodies in $\mathbb{R}^{n}$, i.e., the family of all non-empty convex and compact subsets $K \subset \mathbb{R}^{n}$. We write $B_{2}^{n}$ for the $n$-dimensional Euclidean unit ball and its boundary bd $B_{2}^{n}$ is denoted by $\mathbb{S}^{n-1}$. The volume of $X \subset \mathbb{R}^{n}$, i.e., its $n$-dimensional Lebesgue measure is denoted by $|X|$, or by $|X|_{n}$ if the distinction of the dimension is needed.

At the heart of the classical Brunn-Minkowski theory is the study of the volume functional with respect to the Minkowski sum of convex bodies. This leads to the theory of mixed volumes and, in particular, to the (relative) quermassintegrals $\mathrm{W}_{i}(K, L)$ of two convex bodies $K, L \in \mathcal{K}^{n}$. The latter may be defined via the classical (relative) Steiner polynomial, expressing the volume of the Minkowski sum

$$
K+\lambda L=\{x+\lambda y: x \in K, y \in L\},
$$

2000 Mathematics Subject Classification. Primary 52A30, 52A39; Secondary 30C15.

Key words and phrases. Dual quermassintegrals, dual Steiner polynomials, Shephard's problem, moment problem, location of roots.

Supported by: MINECO/FEDER projects MTM2016-77710-P and MTM2015-65430P; "Programa de Ayudas a Grupos de Excelencia de la Región de Murcia", Fundación Séneca, 19901/GERM/15. 
$\lambda \geq 0$, as a polynomial in $\lambda$ (cf., e.g., [25, s. 5.1], [27]):

$$
|K+\lambda L|=\sum_{i=0}^{n}\left(\begin{array}{l}
n \\
i
\end{array}\right) \mathrm{W}_{i}(K, L) \lambda^{i} .
$$

In particular, we have $\mathrm{W}_{0}(K, L)=|K|$ and $\mathrm{W}_{n}(K, L)=|L|$. The other quermassintegrals admit in general not such a direct and simple geometric interpretation. For $L=B_{2}^{n}$, however, we have Kubota's integral formula (cf., e.g., [25, (5.72)]),

$$
\mathrm{W}_{n-i}\left(K, B_{2}^{n}\right)=\left.\frac{\left|B_{2}^{n}\right|}{\left|B_{2}^{i}\right|_{i}} \int_{G(n, i)}|K| E\right|_{i} \mathrm{~d} E, \quad i=1, \ldots, n,
$$

where integration is taken with respect to the rotation-invariant probability measure on the Grassmannian $G(n, i)$ of all $i$-dimensional linear subspaces $E \subset \mathbb{R}^{n}$, and $K \mid E$ denotes the image of the orthogonal projection onto $E$. Hence, the quermassintegrals are (up to some constants) the means of the volumes of projections.

There are two far-reaching extensions of the classical Brunn-Minkowski theory, both arising basically by replacing the classical Minkowski (vectorwise) addition by another additive operation (cf. [8, 9]), and both being cornerstones of modern convex geometry. The first one is the $L_{p}$-addition, introduced and studied by Firey in [5]. A systematic investigation of the $L_{p}$-sum of convex bodies and its consequences was started by Lutwak in [20, 21]. It led to the rich and emerging $L_{p}$-Brunn-Minkowski theory for which we refer to $[25$, s. 9.1,9.2]).

The second one, the dual Brunn-Minkowski theory, was introduced by Lutwak in [18, 19], and is based on the radial addition $x \widetilde{+} y$ for $x, y \in \mathbb{R}^{n}$, where

$$
x \widetilde{+} y= \begin{cases}x+y, & \text { if } x, y \text { are linearly dependent }, \\ 0, & \text { otherwise. }\end{cases}
$$

In general, the radial sum $K \widetilde{+} L=\{x \widetilde{+} y: x \in K, y \in L\}$ of two convex bodies $K, L$ is not a convex set, but the radial sum of two star bodies is again a star body. In fact, the dual Brunn-Minkowski theory operates on this (larger) class of sets, and this is also one of the features of the dual Brunn-Minkowski theory which makes it so useful. The celebrated solution of the Busemann-Petty problem is amongst the recent successes of the dual Brunn-Minkowski theory (cf. 6, 10, 28), and it also has connections and applications to integral geometry, Minkowski geometry and the local theory of Banach spaces.

In order to define star bodies, we call a non-empty set $S \subseteq \mathbb{R}^{n}$ starshaped (with respect to the origin) if the segment $[0, x] \subseteq S$ for all $x \in S$. For a compact starshaped set $K$ its radial function $\rho_{K}: \mathbb{S}^{n-1} \longrightarrow \mathbb{R}_{\geq 0}$ is defined by

$$
\rho_{K}(u)=\max \{\rho \geq 0: \rho u \in K\} .
$$

If this function is positive and continuous then $K$ is called a star body. In particular, any star body has non-empty interior and any convex body 
containing the origin in its interior is a star body. Let $\mathcal{S}_{0}^{n}$ be the set of all star bodies in $\mathbb{R}^{n}$. Considering now the volume of the radial sum $K \tilde{+} \lambda L$ for $K, L \in \mathcal{S}_{0}^{n}$ and $\lambda \geq 0$, i.e.,

$$
K \widetilde{+} \lambda L=\{x \widetilde{+} \lambda y: x \in K, y \in L\},
$$

leads, in analogy to the classical Brunn-Minkowski theory, to the (relative) dual Steiner polynomial (see e.g. [18], [25, p. 508]),

$$
|K \widetilde{+} \lambda L|=\sum_{i=0}^{n}\left(\begin{array}{c}
n \\
i
\end{array}\right) \widetilde{\mathrm{W}}_{i}(K, L) \lambda^{i} .
$$

The coefficients $\widetilde{\mathrm{W}}_{i}(K, L)$ are the (relative) dual quermassintegrals of $K$ and $L$. They are special cases of the dual mixed volumes, which were introduced by Lutwak in [18] (see also [25, s. 9.3]). As in the classical case, we have $\widetilde{\mathrm{W}}_{0}(K, L)=|K|$ and $\widetilde{\mathrm{W}}_{n}(K, L)=|L|$. Moreover, in analogy to Kubota's formula (1.2), the dual quermassintegrals $\widetilde{\mathrm{W}}_{i}\left(K, B_{2}^{n}\right)$ admit an integral geometric representation as the means of the volumes of sections (cf. [25, (9.38)]):

$$
\widetilde{\mathrm{W}}_{n-i}\left(K, B_{2}^{n}\right)=\frac{\left|B_{2}^{n}\right|}{\left|B_{2}^{i}\right|_{i}} \int_{G(n, i)}|K \cap E|_{i} \mathrm{~d} E, \quad i=1, \ldots, n .
$$

There are many more "dualities" and similarities between the classical and the dual theory. In this context, as well as for the immense impact of the dual Brunn-Minkowski theory, we refer, for instance, to [1, 2, 4, 7, 14, 16, 25] and the references inside.

In contrast to the classical quermassintegrals, the dual quermassintegrals have a direct integral geometric representation in terms of the radial functions of the involved star bodies $K, L \in \mathcal{S}_{0}^{n}$ ([18], [25, s. 9.3]):

$$
\widetilde{\mathrm{W}}_{i}(K, L)=\frac{1}{n} \int_{\mathbb{S}^{n-1}} \rho_{K}(u)^{n-i} \rho_{L}(u)^{i} \mathrm{~d} \sigma(u) .
$$

Here $\sigma$ is the usual spherical Lebesgue measure. We also observe that (1.4) defines dual quermassintegrals $\widetilde{\mathrm{W}}_{i}(K, L)$ for any real index $i \in \mathbb{R}$, whereas $\mathrm{W}_{i}(K, L)$, for $K, L \in \mathcal{K}^{n}$, is only defined for $i=0, \ldots, n$.

Given a polynomial associated to a geometric (or combinatorial, algebraic, etc.) structure, it is a natural and central problem to characterize these polynomials among all polynomials of a given degree, i.e., to find necessary and sufficient conditions determining the set of coefficients of the polynomials in question.

Regarding the Steiner polynomial (1.1), this characterization problem was solved by Shephard in [26]. He proved that the well-known AleksandrovFenchel inequalities (see [25, (9.40)]) are necessary and sufficient conditions to characterize the quermassintegrals of two convex bodies (see also [13]). 
For general mixed volumes the problem is open. More precisely, given $k \geq 2$ convex bodies $K_{1}, \ldots, K_{k} \in \mathcal{K}^{n}$, there are $N=\left(\begin{array}{c}n+k-1 \\ n\end{array}\right)$ mixed volumes $\mathrm{V}\left(K_{i_{1}}, \ldots, K_{i_{n}}\right), 1 \leq i_{1} \leq \cdots \leq i_{n} \leq k$, where we refer to [25, s. 5.1] for a thorough study of mixed volumes. A set of inequalities is called a full set if for given $N$ (non-negative) numbers satisfying these inequalities, there exist $k$ convex bodies whose mixed volumes are the given numbers. In this terminology, Shephard proved that for $k=2$ the Aleksandrov-Fenchel inequalities form a full set. He also showed that if $k=n+2$ the known inequalities are not a full set. Moreover, for $k=3$ and $n=2$, Heine [12] proved that the Aleksandrov-Fenchel inequalities together with the determinantal inequality $\operatorname{det}\left(\mathrm{V}\left(K_{i}, K_{j}\right)_{1 \leq i, j \leq 3}\right) \geq 0$ yield a full set. Even for more than three planar convex bodies the problem is open.

The main aim of this paper is to present an analogue of Shephard's result within the dual Brunn-Minkowski theory, i.e., to characterize dual quermassintegrals of star bodies. The first substantial difference to the classical case is that dual quermassintegrals are defined for any real index (cf. (1.4) independently of the dimension of the involved star bodies. Therefore, we consider here tuples of finitely many distinct real numbers (indices) $\left(r_{0}=0, r_{1}, \ldots, r_{m}\right), r_{j} \in \mathbb{R}, 1 \leq j \leq m$, and for $n \geq 2$ we give a characterization of those tuples $\left(\omega_{0}, \omega_{1}, \ldots, \omega_{m}\right)$ of positive real numbers $\omega_{i}$ such that there exist $n$-dimensional star bodies $K, L$ with $\widetilde{\mathrm{W}}_{r_{j}}(K, L)=\omega_{j}$, $j=0, \ldots, m$.

We note that we have to assume that 0 appears as index, meaning that we need the information about the volume of $K$, since $|K|=\widetilde{\mathrm{W}}_{0}(K, L)=\omega_{0}$. Moreover, throughout the paper we will always assume that such a tuple $\left(r_{0}, r_{1}, \ldots, r_{m}\right)$ consists of pairwise distinct numbers.

In order to state our characterization we need a bit more notation. We write int $X$ to represent the interior of a set $X \subseteq \mathbb{R}^{n}$, and we use conv $X$ and pos $X$ for its convex and positive hulls, respectively.

For $0<a<b$ and for $R=\left(r_{0}=0, r_{1}, \ldots, r_{m}\right), r_{j} \in \mathbb{R}, 1 \leq j \leq m$, we write

$$
C_{a, b}^{R}=\operatorname{pos}\left\{\left(t^{r_{0}}=1, t^{r_{1}}, t^{r_{2}}, \ldots, t^{r_{m}}\right)^{\top}: t \in[a, b]\right\} \subset \mathbb{R}^{m+1} .
$$

Theorem 1.1. Let $R=\left(r_{0}=0, r_{1}, \ldots, r_{m}\right), r_{j} \in \mathbb{R}, 1 \leq j \leq m$, let $\omega_{i} \in \mathbb{R}_{>0}, 0 \leq i \leq m$, be positive real numbers and let $n \geq 2$. Then there exist star bodies $K, L \in \mathcal{S}_{0}^{n}$ such that

$$
\widetilde{\mathrm{W}}_{r_{i}}(K, L)=\omega_{i}, \quad 0 \leq i \leq m,
$$

if and only if

i) either there exist $0<a<b$ such that

$$
\left(\omega_{0}, \omega_{1}, \ldots, \omega_{m}\right)^{\top} \in \operatorname{int} C_{a, b}^{R},
$$

ii) or $\omega_{i}=\lambda^{r_{i}} \omega_{0}$ for some $\lambda>0$ and $1 \leq i \leq m$. 
Observe, that the dimension $n$ of the star bodies can be freely chosen, and that the second case ii) corresponds to $L=\lambda K$ (cf. Lemma 2.1 ii) in Section 2). We also want to point out that item i) is equivalent to the fact that $\left(\omega_{0}, \omega_{1}, \ldots, \omega_{m}\right)^{\top} \in \operatorname{int} \operatorname{pos}\left\{\left(t^{r_{0}}=1, t^{r_{1}}, t^{r_{2}}, \ldots, t^{r_{m}}\right)^{\top}: t>0\right\}$ (see Remark 2.2). For our purposes, however, the formulation as in item i) is more suitable, e.g., for Theorem 2.2 , in which we provide another characterization of the dual quermassintegrals $\widetilde{W}_{i}(K, L)$ for natural indices $i=0, \ldots, m$, via the positive definiteness of certain Hankel-matrices.

The characterization of dual quermassintegrals in Theorem 1.1 is related to the moment problem (see e.g. [17]). Based on this relation, we will also get new determinantal inequalities between dual quermassintegrals, proved in Section 3, The main result is here:

Theorem 1.2. Let $K, L \in \mathcal{S}_{0}^{n}$ and let $m \in \mathbb{N}, m \geq 1$. For pairwise distinct numbers $r_{1}, \ldots, r_{m} \in \mathbb{R}$, let $A_{m} \in \mathbb{R}^{m \times m}$ be the Hankel matrix

$$
A_{m}=\left(\widetilde{\mathrm{W}}_{r_{i}+r_{j}}(K, L)\right)_{1 \leq i, j \leq m} .
$$

Then $\operatorname{det} A_{m} \geq 0$ with equality if and only if $K=\lambda L$ for some $\lambda>0$.

As a corollary we get, for instance, the following result:

Corollary 1.1. Let $K, L \in \mathcal{S}_{0}^{n}$. For $m \in \mathbb{N}$ let

$$
\Delta_{m}=\left(\begin{array}{cccc}
\widetilde{\mathrm{W}}_{0}(K, L) & \widetilde{\mathrm{W}}_{1}(K, L) & \cdots & \widetilde{\mathrm{W}}_{m}(K, L) \\
\widetilde{\mathrm{W}}_{1}(K, L) & \widetilde{\mathrm{W}}_{2}(K, L) & \cdots & \widetilde{\mathrm{W}}_{m+1}(K, L) \\
\vdots & \vdots & \vdots & \vdots \\
\widetilde{\mathrm{W}}_{m}(K, L) & \widetilde{\mathrm{W}}_{m+1}(K, L) & \cdots & \widetilde{\mathrm{W}}_{2 m}(K, L)
\end{array}\right) .
$$

Then det $\Delta_{m} \geq 0$ with equality if and only if $K=\lambda L$ for some $\lambda>0$.

As pointed out in Remark 3.1, these determinantal inequalities yield new inequalities for the dual quermassintegrals. In the classical setting, the validity of a family of determinantal inequalities for mixed volumes remains an open problem (see [3]).

In several recent articles (see, e.g., 13 and the references therein), the characterization of the quermassintegrals of convex bodies became a key tool in order to study structural properties of the set of roots of the relative Steiner polynomial, regarded as a polynomial in a complex variable, cf. (1.1).

With the help of Theorem 1.1, we also carry out the corresponding study for the roots of dual Steiner polynomials. To this end we regard the right hand side in $(1.3)$ as a formal polynomial in a complex variable $z \in \mathbb{C}$, which we denote by

$$
\widetilde{f}_{K, L}(z)=\sum_{i=0}^{n}\left(\begin{array}{c}
n \\
i
\end{array}\right) \widetilde{\mathrm{W}}_{i}(K, L) z^{i} .
$$

We observe that 0 cannot be a root of any dual Steiner polynomial because $\widetilde{\mathrm{W}}_{0}(K, L)=|K|>0$ for $K \in \mathcal{S}_{0}^{n}$. 
Here we are interested in the location and the structure of the roots of $\widetilde{f}_{K, L}(z)$. To this end, let $\mathbb{C}^{+}=\{z \in \mathbb{C}: \operatorname{Im}(z) \geq 0\}$, and for $n \geq 2$ let

$$
\widetilde{\mathcal{R}}(n)=\left\{z \in \mathbb{C}^{+}: \widetilde{f}_{K, L}(z)=0 \text { for some } K, L \in \mathcal{S}_{0}^{n}\right\}
$$

be the set of all roots of all dual Steiner polynomials in the upper half-plane. We prove the following result.

Theorem 1.3. Let $n \geq 1$.

a) $\widetilde{\mathcal{R}}(n)$ is a convex cone containing the negative real axis $\mathbb{R}_{<0}$.

b) $\widetilde{\mathcal{R}}(n) \cap \operatorname{bd} \widetilde{\mathcal{R}}(n)=\mathbb{R}_{<0}$, i.e., it is half-open.

c) $\widetilde{\mathcal{R}}(n)$ is monotonous in the dimension, i.e., $\widetilde{\mathcal{R}}(n) \subseteq \widetilde{\mathcal{R}}(n+1)$.

In view of [13, Theorem 1.1 and Theorem 1.3] we note that the dual Steiner polynomial shares properties a) and c) with the classical Steiner polynomial. However, property b) provides a first structural difference between both polynomials, since the cone of roots of the classical Steiner polynomial is closed (see [13, Theorem 1.2]).

The above theorem will be proved in Section 4, along with several additional properties of the roots. In Section 2 we give characterizations of dual quermassintegrals of star bodies, in particular, the proof of Theorem 1.1 . The proof of Theorem 1.2 as well as further determinantal inequalities are contained in Section 3 .

\section{Characterization of Dual Quermassintegrals}

For any $r \in \mathbb{R}$, the $r$-th dual quermassintegral is a monotonous and homogeneous functional of degree $n-r$ in its first argument and of degree $r$ in the second one (cf. (1.4)), i.e., given $K, K^{\prime}, L \in \mathcal{S}_{0}^{n}$ with $K \subseteq K^{\prime}, \lambda>0$, and $r \in \mathbb{R}$, then

$$
\begin{aligned}
\widetilde{\mathrm{W}}_{r}(K, L) & \leq \widetilde{\mathrm{W}}_{r}\left(K^{\prime}, L\right) \quad \text { and } \\
\widetilde{\mathrm{W}}_{r}(\lambda K, L) & =\lambda^{n-r} \widetilde{\mathrm{W}}_{r}(K, L), \quad \widetilde{\mathrm{W}}_{r}(K, \lambda L)=\lambda^{r} \widetilde{\mathrm{W}}_{r}(K, L) .
\end{aligned}
$$

It is also well-known that for $K, L \in \mathcal{S}_{0}^{n}$ (see e.g. [25, (9.40)])

$$
\widetilde{\mathrm{W}}_{s}(K, L)^{t-r} \leq \widetilde{\mathrm{W}}_{r}(K, L)^{t-s} \widetilde{\mathrm{W}}_{t}(K, L)^{s-r}, \quad r<s<t,
$$

which are the "dual" counterpart to the classical Aleksandrov-Fenchel inequalities, but now with real $r, s, t$. In (2.1) equality holds if and only if $K$ and $L$ are dilates.

The next lemma collects two more basic properties of dual quermassintegrals which will be needed later.

Lemma 2.1. Let $K, L \in \mathcal{S}_{0}^{n}$.

i) If $L \subseteq K$ and $r<s$ then

$$
\widetilde{\mathrm{W}}_{r}(K, L) \geq \widetilde{\mathrm{W}}_{s}(K, L) .
$$

ii) $\widetilde{\mathrm{W}}_{\alpha}(K, L)=\mu^{\alpha} \widetilde{\mathrm{W}}_{0}(K, L)$ for $\alpha=r, s, t \in \mathbb{R}, r<s<t$, and some $\mu>0$, if and only if $L=\mu K$. 
Proof. For i) we observe that $\rho_{L}(u) \leq \rho_{K}(u)$ for $u \in \mathbb{S}^{n-1}$ and hence, by (1.4),

$$
\begin{aligned}
\widetilde{\mathrm{W}}_{r}(K, L) & =\frac{1}{n} \int_{\mathbb{S}^{n-1}} \rho_{K}(u)^{n-r} \rho_{L}(u)^{r} \mathrm{~d} \sigma(u) \\
& =\frac{1}{n} \int_{\mathbb{S}^{n-1}} \rho_{K}(u)^{n-s} \rho_{L}(u)^{s}\left(\frac{\rho_{K}(u)}{\rho_{L}(u)}\right)^{s-r} \mathrm{~d} \sigma(u) \\
& \geq \frac{1}{n} \int_{\mathbb{S}^{n-1}} \rho_{K}(u)^{n-s} \rho_{L}(u)^{s} \mathrm{~d} \sigma(u)=\widetilde{\mathrm{W}}_{s}(K, L) .
\end{aligned}
$$

In order to verify ii) we note that $\widetilde{\mathrm{W}}_{\alpha}(K, L)=\mu^{\alpha} \widetilde{\mathrm{W}}_{0}(K, L)$ for $\alpha=r, s, t$ implies

$$
\widetilde{\mathrm{W}}_{r}(K, L)^{t-s} \widetilde{\mathrm{W}}_{t}(K, L)^{s-r}=\mu^{s(t-r)} \widetilde{\mathrm{W}}_{0}(K, L)^{t-r}=\widetilde{\mathrm{W}}_{s}(K, L)^{t-r} .
$$

Hence, we have equality in (2.1), which yields $L=\mu K$. The converse is obviously true by the homogeneity of the dual quermassintegrals.

For the proof of Theorem 1.1 we need the following notation: given a measure $\mu$ on an interval $[a, b] \subset(0, \infty)$ we write, for any $r \in \mathbb{R}$,

$$
m_{r}(\mu)=\int_{a}^{b} t^{r} \mathrm{~d} \mu(t) .
$$

When $r \in \mathbb{N} \cup\{0\}$, the above numbers are the moments of $\mu$ on the interval $[a, b]$ and, roughly speaking, the existence of a measure with prescribed moments is the so-called moment problem. For a thorough study we refer, e.g., to [17, 24].

A quite general answer to the existence problem of such a measure on a finite interval $[a, b]$ is provided by the following refinement of Riesz's representation theorem (see, e.g., [17, Theorem 3.5 and P. 3.9 in p. 17]). It will be also a key tool in our proof of Theorem 1.1 and provides the connection between our characterization of dual quermassintegrals and the moment problem.

Theorem 2.1 (Riesz). Let $\alpha:[a, b] \longrightarrow \mathbb{R}^{n}, \alpha(t)=\left(\alpha_{1}(t), \ldots, \alpha_{n}(t)\right)^{\top}$, be a curve in $\mathbb{R}^{n}$ not contained in a hyperplane through 0 , and let $x \in \mathbb{R}^{n}$. There exists a probability measure $\mu$ on $[a, b]$ such that

$$
x_{i}=\int_{a}^{b} \alpha_{i}(t) \mathrm{d} \mu(t), \quad \text { for every } i=1, \ldots n,
$$

if and only if $x \in \operatorname{conv}\{\alpha(t): t \in[a, b]\}$.

Moreover, $x \in \operatorname{int} \operatorname{conv}\{\alpha(t): t \in[a, b]\}$ if and only if there exits a continuous function $\phi:[a, b] \longrightarrow(0, \infty)$ such that $\mathrm{d} \mu(t)=\phi(t) \mathrm{d} t$.

As a corollary we deduce that the above property regarding interior points is equivalent to the fact that the measure $\mu$ can be assumed to be supported on the whole interval $[a, b]$. 
Corollary 2.1. Let $\alpha:[a, b] \longrightarrow \mathbb{R}^{n}$ be a continuous curve in $\mathbb{R}^{n}$ not contained in a hyperplane through 0 , and let $x \in \mathbb{R}^{n}$. There exists a probability measure $\mu$ on $[a, b]$ such that $\mu([c, d))>0$ for every $[c, d) \subset[a, b]$ and

$$
x_{i}=\int_{a}^{b} \alpha_{i}(t) \mathrm{d} \mu(t), \quad \text { for every } i=1, \ldots n,
$$

if and only if $x \in \operatorname{int} \operatorname{conv}\{\alpha(t): t \in[a, b]\}$.

Proof. First we suppose that $x \in \operatorname{int} \operatorname{conv}\{\alpha(t): t \in[a, b]\}$. Then, by Theorem 2.1, there exists a probability measure $\mu$ satisfying (2.3) and with a positive density $\phi$ with respect to the Lebesgue measure. Thus $\mu([c, d))>0$ for all $[c, d) \subset[a, b]$.

Conversely, if we suppose the existence of a measure $\mu$ satisfying our assumptions, Theorem 2.1 gives $x \in \operatorname{conv}\{\alpha(t): t \in[a, b]\}$. So, let us assume that $x \notin \operatorname{int} \operatorname{conv}\{\alpha(t): t \in[a, b]\}$. Then there exists a supporting hyperplane to $\operatorname{conv}\{\alpha(t): t \in[a, b]\}$ at $x$ with outer normal vector $u \in \mathbb{S}^{n-1}$, say, and $\langle y, u\rangle \leq\langle x, u\rangle$ for $y \in \operatorname{conv}\{\alpha(t): t \in[a, b]\}$. Furthermore, since $\alpha([a, b])$ is not contained in a hyperplane, there exists $[c, d) \subset[a, b]$ with $\langle\alpha(t), u\rangle<\langle x, u\rangle$ for $t \in[c, d)$. Thus, we get the contradiction

$$
\langle x, u\rangle=\int_{a}^{b}\langle\alpha(t), u\rangle \mathrm{d} \mu(t)<\langle x, u\rangle,
$$

and so $x \in \operatorname{int} \operatorname{conv}\{\alpha(t): t \in[a, b]\}$.

The next lemma finally relates the dual quermassintegrals to the real moments 2.2 .

Lemma 2.2. Let $[a, b] \subset(0, \infty)$, $a<b$, let $n, m \geq 2$, and let $r_{j} \in \mathbb{R}$, $j=1, \ldots, m$, and $r_{0}=0$. Let $\mu$ be a positive measure on $[a, b]$ such that $\mu([c, d))>0$ for every $[c, d) \subset[a, b]$ and $\mu([a, b])=\left|B_{2}^{n}\right|$. Then there exists $L \in \mathcal{S}_{0}^{n}$ satisfying

$$
\widetilde{\mathrm{W}}_{r_{j}}\left(B_{2}^{n}, L\right)=m_{r_{j}}(\mu), \quad 0 \leq j \leq m .
$$

Proof. Let $F:[a, b] \longrightarrow[0,1]$ be the function defined by

$$
F(t)=\frac{\mu([t, b])}{\mu([a, b])} .
$$

Our assumptions ensure that $F$ is a strictly decreasing function, continuous from the left and satisfies $F(a)=1$ and $F(b)=\mu(\{b\}) / \mu([a, b])$. Let $G:[0,1] \longrightarrow[a, b]$ be the function

$$
G(s)=\sup \{t \in[a, b]: F(t) \geq s\},
$$

which coincides with $F^{-1}$ when $F$ is bijective. Since $F$ is strictly decreasing, it is easy to see that $G$ is decreasing and continuous, and so, the function 
$\rho_{L}: \mathbb{S}^{n-1} \longrightarrow[a, b]$ given by

$$
\rho_{L}(u)=G\left(\frac{\sigma\left(\left\{v \in \mathbb{S}^{n-1}:\left|v_{1}\right| \geq\left|u_{1}\right|\right\}\right)}{\sigma\left(\mathbb{S}^{n-1}\right)}\right)
$$

is continuous and positive on $\mathbb{S}^{n-1}$. Hence, it defines a star body $L \in \mathcal{S}_{0}^{n}$. Moreover, since $\rho_{L}(w) \geq \rho_{L}(u)$ if and only if $\left|w_{1}\right| \geq\left|u_{1}\right|$, we get for $t \in[a, b]$

$$
\frac{\sigma\left(\left\{v \in \mathbb{S}^{n-1}: \rho_{L}(v) \geq t\right\}\right)}{\sigma\left(\mathbb{S}^{n-1}\right)}=F(t)=\frac{\mu([t, b])}{\mu([a, b])} .
$$

In the case $t \notin[a, b]$ we trivially have

$$
\frac{\sigma\left(\left\{v \in \mathbb{S}^{n-1}: \rho_{L}(v) \geq t\right\}\right)}{\sigma\left(\mathbb{S}^{n-1}\right)}=\frac{\mu(\{s \in[a, b]: s \geq t\})}{\mu([a, b])} .
$$

Finally, since $\mu([a, b])=m_{0}(\mu)=\left|B_{2}^{n}\right|$ we have $\widetilde{\mathrm{W}}_{r_{0}}\left(B_{2}^{n}, L\right)=m_{0}(\mu)$, and since $\sigma\left(\mathbb{S}^{n-1}\right)=n\left|B_{2}^{n}\right|$, we find by the layer cake formula for $j=1, \ldots, m$,

$$
\begin{aligned}
\widetilde{\mathrm{W}}_{r_{j}}\left(B_{2}^{n}, L\right) & =\frac{1}{n} \int_{\mathbb{S}^{n-1}} \rho_{L}^{r_{j}}(u) \mathrm{d} \sigma(u) \\
& =\frac{1}{n} \int_{0}^{\infty} r_{j} t^{r_{j}-1} \sigma\left(\left\{v \in \mathbb{S}^{n-1}: \rho_{L}(v) \geq t\right\}\right) \mathrm{d} t \\
& =\frac{\left|B_{2}^{n}\right|}{\mu([a, b])} \int_{0}^{\infty} r_{j} t^{r_{j}-1} \mu(\{s \in[a, b]: s \geq t\}) \mathrm{d} t \\
& =\int_{a}^{b} s^{r_{j}} \mathrm{~d} \mu(s)=m_{r_{j}}(\mu) .
\end{aligned}
$$

Now we are ready to prove Theorem 1.1 .

Proof of Theorem 1.1. We start assuming the existence of star bodies $K, L \in$ $\mathcal{S}_{0}^{n}$ such that $\widetilde{\mathrm{W}}_{r_{i}}(K, L)=\omega_{i}$ for $i=0, \ldots, m$. We denote by $f: \mathbb{S}^{n-1} \longrightarrow$ $(0, \infty)$ the continuous function $f(u)=\rho_{L}(u) / \rho_{K}(u)$, and let

$$
a=\min _{u \in \mathbb{S}^{n-1}} f(u) \text { and } b=\max _{u \in \mathbb{S}^{n-1}} f(u) .
$$

Let $\nu$ be the measure on the sphere given by $\mathrm{d} \nu=(1 / n) \rho_{K}^{n}(u) \mathrm{d} \sigma(u)$ and let $\mu$ be the push-forward measure of $\nu$ by $f$. Then $\mu$ is supported on $[a, b]$ and it is defined by

$$
\mu(A)=\nu\left(f^{-1}(A)\right)=\frac{1}{n} \int_{f^{-1}(A)} \rho_{K}^{n}(u) \mathrm{d} \sigma(u)
$$


for any Borel subset $A \subseteq[a, b]$. Consequently, in the case $a<b$ we have

$$
\begin{aligned}
\int_{a}^{b} t^{r_{i}} \mathrm{~d} \mu(t) & =\int_{\mathbb{S}^{n-1}} f(u)^{r_{i}} \mathrm{~d} \nu(u) \\
& =\frac{1}{n} \int_{\mathbb{S}^{n-1}}\left(\frac{\rho_{L}(u)}{\rho_{K}(u)}\right)^{r_{i}} \rho_{K}^{n}(u) \mathrm{d} \sigma(u) \\
& =\widetilde{\mathrm{W}}_{r_{i}}(K, L)=\omega_{i}, \quad i=0, \ldots, m
\end{aligned}
$$

(cf. (1.4)). The measure $\mathrm{d} \mu / \widetilde{\mathrm{W}}_{0}(K, L)$ is a probability measure with support $[a, b]$. Then, the second part in Theorem 2.1 applied to the "real moment curve" $\alpha(t)=\left(t^{r_{1}}, t^{r_{2}}, \ldots, t^{r_{m}}\right)^{\top}$ ensures that

$$
\left(\frac{\omega_{1}}{\omega_{0}}, \frac{\omega_{2}}{\omega_{0}}, \ldots, \frac{\omega_{m}}{\omega_{0}}\right)^{\top} \in \operatorname{int} \operatorname{conv}\left\{\left(t^{r_{1}}, t^{r_{2}}, \ldots, t^{r_{m}}\right)^{\top}: t \in[a, b]\right\}
$$

and hence

$$
\left(\omega_{0}, \omega_{1}, \ldots, \omega_{m}\right)^{\top} \in \operatorname{int} C_{a, b}^{R} .
$$

On the other hand, if $a=b$ then $f(u)=a$ for every $u \in \mathbb{S}^{n-1}$, which implies that $L=a K$, and hence $\omega_{i}=\widetilde{\mathrm{W}}_{r_{i}}(K, L)=a^{r_{i}}|K|=a^{r_{i}} \omega_{0}$ for $i=0, \ldots, m$.

For the converse direction, let $0<a<b$ and

$$
\left(\omega_{0}, \omega_{1}, \ldots, \omega_{m}\right)^{\top} \in \operatorname{int} C_{a, b}^{R} .
$$

Then

$$
\left(\frac{\omega_{1}}{\omega_{0}}, \frac{\omega_{2}}{\omega_{0}}, \ldots, \frac{\omega_{m}}{\omega_{0}}\right)^{\top} \in \operatorname{int} \operatorname{conv}\left\{\left(t^{r_{1}}, t^{r_{2}}, \ldots, t^{r_{m}}\right)^{\top}: t \in[a, b]\right\},
$$

and Corollary 2.1 yields the existence of a probability measure $\mu$ on $[a, b]$ such that $\mu([c, d))>0$ for every $[c, d) \subset[a, b]$ and

$$
\frac{\omega_{i}}{\omega_{0}}=\int_{a}^{b} t^{r_{i}} \mathrm{~d} \mu(t), \quad i=0, \ldots, m
$$

Hence, since

$$
m_{r_{i}}\left(\left|B_{2}^{n}\right| \mu\right)=\int_{a}^{b} t^{r_{i}}\left|B_{2}^{n}\right| \mathrm{d} \mu(t)=\frac{\left|B_{2}^{n}\right| \omega_{i}}{\omega_{0}},
$$

application of Lemma 2.2 to the measure $\left|B_{2}^{n}\right| \mu$ implies the existence of a star body $L^{\prime} \in \mathcal{S}_{0}^{n}$ such that for $i=0, \ldots, m$

$$
\frac{\left|B_{2}^{n}\right| \omega_{i}}{\omega_{0}}=\widetilde{\mathrm{W}}_{r_{i}}\left(B_{2}^{n}, L^{\prime}\right)
$$

Thus, with

$$
K=\left(\frac{\omega_{0}}{\left|B_{2}^{n}\right|}\right)^{1 / n} B_{2}^{n} \quad \text { and } \quad L=\left(\frac{\omega_{0}}{\left|B_{2}^{n}\right|}\right)^{1 / n} L^{\prime},
$$

we get, for $i=0, \ldots, m$,

$$
\omega_{i}=\widetilde{\mathrm{W}}_{r_{i}}(K, L)
$$


Finally, if there exists $\lambda>0$ such that $\omega_{i}=\lambda^{r_{i}} \omega_{0}, 0 \leq i \leq m$, any star body $K \in \mathcal{S}_{0}^{n}$ with $|K|=\omega_{0}$ and $L=\lambda K$ yield $\omega_{i}=\widetilde{\mathrm{W}}_{r_{i}}(K, L)$.

Remark 2.1. We want to point out that the proof above, in particular, shows that for non-homothetic $K, L \in \mathcal{S}_{0}^{n}$ (cf. (2.4), (2.5)

$$
\left(\widetilde{\mathrm{W}}_{r_{0}}(K, L), \ldots, \widetilde{\mathrm{W}}_{r_{m}}(K, L)\right)^{\top} \in \operatorname{int} C_{a, b}^{R}
$$

with $a=\min _{u \in \mathbb{S}^{n-1}} \rho_{L}(u) / \rho_{K}(u)$ and $b=\max _{u \in \mathbb{S}^{n-1}} \rho_{L}(u) / \rho_{K}(u)$.

Remark 2.2. We note that item i) in Theorem 1.1 is equivalent to

$$
\left(\omega_{0}, \omega_{1}, \ldots, \omega_{m}\right)^{\top} \in \operatorname{int} \operatorname{pos}\left\{\left(t^{r_{0}}=1, t^{r_{1}}, t^{r_{2}}, \ldots, t^{r_{m}}\right)^{\top}: t>0\right\}=: \operatorname{int} C^{R} .
$$

Indeed, clearly $\operatorname{int} C_{a, b}^{R} \subset \operatorname{int} C^{R}$ for any $0<a<b$. On the other hand, if $x \in \operatorname{int} C^{R}$ then Steinitz's theorem (see e.g. [25, Theorem 1.3.10]) implies that there exist $t_{1}, \ldots, t_{k} \in \mathbb{R}_{>0}$, with $k=2(m+1)$, such that

$$
x \in \operatorname{int} \operatorname{pos}\left\{\left(1, t_{1}^{r_{1}}, \ldots, t_{1}^{r_{m}}\right)^{\top}, \ldots,\left(1, t_{k}^{r_{1}}, \ldots, t_{k}^{r_{m}}\right)^{\top}\right\} .
$$

With $a=\min \left\{t_{1}, \ldots, t_{k}\right\}$ and $b=\max \left\{t_{1}, \ldots, t_{k}\right\}$ we get $x \in \operatorname{int} C_{a, b}^{R}$.

Obviously, in order to apply Theorem 1.1 it would convenient to have a more explicit description of the cone $C_{a, b}^{R}$, for instance, by "few" polynomial inequalities. For a representation by an infinite family of linear inequalities in the special case $R=(0,1, \ldots, m)$ we refer to [23]. Actually, [23] gives a description of the so-called cyclic body $\operatorname{conv}\left\{\left(t^{1}, t^{2}, \ldots, t^{m}\right): t \in[a, b]\right\}$ as intersection of halfspaces, but, of course, $C_{a, b}^{R}$ is just the conification of the cyclic body (cf. (1.5)).

For the particular sequence of exponents $R=(0,1, \ldots, m)$ we will write $C_{a, b}^{m}$ instead of $C_{a, b}^{R}$ in order to stress its dimension. For these special exponents we will derive from Theorem 1.1 another characterization of the dual quermassintegrals. To this end the following known fact is needed.

Lemma 2.3 ([17, Theorem 1.1 in ch. 3]). Let $0<a<b$ and $m \in \mathbb{N}$. Then $\left(x_{0}, \ldots, x_{m}\right) \in \operatorname{int} C_{a, b}^{m}$ if and only if, for every polynomial $P(t)=\sum_{i=0}^{m} c_{i} t^{i}$, $c_{i} \in \mathbb{R}, P(t) \not \equiv 0$, which is non-negative on $[a, b]$, it holds

$$
\sum_{i=0}^{m} c_{i} x_{i}>0 .
$$

Theorem 2.2. Let $m, n \in \mathbb{N}, m, n \geq 2$, and let $\omega_{i}>0, i=0, \ldots, m$. Then there exist star bodies $K, L \in \mathcal{S}_{0}^{n}$ such that

$$
\widetilde{\mathrm{W}}_{i}(K, L)=\omega_{i} \quad \text { for all } i=0, \ldots, m
$$


if and only if, either there exist $0<a<b$ such that the Hankel matrices $\left(a_{j, k}\right)_{j, k=0}^{\lfloor m / 2\rfloor}$ and $\left(b_{j, k}\right)_{j, k=0}^{\lfloor(m-1) / 2\rfloor}$ given by

$$
\begin{aligned}
& a_{j, k}= \begin{cases}\omega_{j+k}, & \text { if } m \text { is even, } \\
\omega_{j+k+1}-a \omega_{j+k}, & \text { if } m \text { is odd, }\end{cases} \\
& b_{j, k}= \begin{cases}(a+b) \omega_{j+k+1}-a b \omega_{j+k}-\omega_{j+k+2}, & \text { if } m \text { is even, } \\
b \omega_{j+k}-\omega_{j+k+1}, & \text { if } m \text { is odd, }\end{cases}
\end{aligned}
$$

are positive definite, or $\omega_{i}=\lambda^{i} \omega_{0}$ for some $\lambda>0$ and $i=1, \ldots, m$; in this case $L=\lambda K$.

Proof. By Theorem 1.1, $\omega_{i}, i=0, \ldots, m$, are the dual quermassintegrals of two star bodies if and only if, either there exist $0<a<b$ such that

$$
\left(\omega_{0}, \omega_{1}, \ldots, \omega_{m}\right) \in \operatorname{int} C_{a, b}^{m},
$$

or $\omega_{i}=\lambda^{i} \omega_{0}$ for some $\lambda>0, i=1, \ldots, m$. Hence, we have to prove that 2.7) is equivalent to the fact that the Hankel matrices $\left(a_{j, k}\right)_{j, k=0}^{l_{a}},\left(b_{j, k}\right)_{j, k=0}^{l_{b}}$ are positive definite, where $l_{a}=\lfloor m / 2\rfloor$ and $l_{b}=\lfloor(m-1) / 2\rfloor$.

First we assume that there are $0<a<b$ such that the Hankel matrices $\left(a_{j, k}\right)_{j, k=0}^{l_{a}},\left(b_{j, k}\right)_{j, k=0}^{l_{b}}$ are positive definite. We are going to use Lemma 2.3 in order to verify $(2.7)$. To this end let $P(t) \not \equiv 0$ be a polynomial of degree $m$, non-negative on $[a, b]$. According to the Markov-Lukács theorem (see e.g. [17, Theorem 2.2 in ch. 3]), $P(t)$ can be written as

$$
P(t)= \begin{cases}\left(\sum_{i=0}^{l_{a}} c_{i} t^{i}\right)^{2}+(b-t)(t-a)\left(\sum_{i=0}^{l_{b}} d_{i} t^{i}\right)^{2}, & \text { if } m \text { is even } \\ (t-a)\left(\sum_{i=0}^{l_{a}} c_{i} t^{i}\right)^{2}+(b-t)\left(\sum_{i=0}^{l_{b}} d_{i} t^{i}\right)^{2}, & \text { if } m \text { is odd }\end{cases}
$$

for some $c_{i}, d_{i} \in \mathbb{R}$. Then, for $m$ even, and by the positive definiteness of the matrices $\left(a_{j, k}\right)_{j, k=0}^{l_{a}},\left(b_{j, k}\right)_{j, k=0}^{l_{b}}$, we get

$$
\begin{aligned}
0 & <\sum_{j, k=0}^{l_{a}} c_{j} c_{k} a_{j, k}+\sum_{j, k=0}^{l_{b}} d_{j} d_{k} b_{j, k} \\
& =\sum_{j, k=0}^{l_{a}} c_{j} c_{k} \omega_{j+k}+\sum_{j, k=0}^{l_{b}} d_{j} d_{k}\left[(a+b) \omega_{j+k+1}-a b \omega_{j+k}-\omega_{j+k+2}\right] .
\end{aligned}
$$

Thus, Lemma 2.3 shows (2.7). The case $m$ odd can be treated in the same way.

Next we assume that 2.7) holds and let $m$ be even with $m=2 p, p \in \mathbb{N}$. Then $l_{a}=p$ and $l_{b}=p-1$.

For any $c_{0}, \ldots, c_{p} \in \mathbb{R}$, not all zero, the polynomial

$$
\left(\sum_{k=0}^{p} c_{k} t^{k}\right)^{2}=\sum_{j, k=0}^{p} c_{j} c_{k} t^{j+k}
$$


is non-negative. Hence, Lemma 2.3 yields

$$
\sum_{j, k=0}^{p} c_{j} c_{k} \omega_{j+k}>0
$$

and thus, the Hankel matrix $\left(\omega_{j+k}\right)_{j, k=0}^{p}$ is positive definite. Analogously, for any $d_{0}, \ldots, d_{p-1} \in \mathbb{R}$, not all zero, the polynomial

$$
(b-t)(t-a)\left(\sum_{k=0}^{p-1} d_{k} t^{k}\right)^{2}=\sum_{j, k=0}^{p-1} d_{j} d_{k}\left[(a+b) t^{j+k+1}-a b t^{j+k}-t^{j+k+2}\right]
$$

is non-negative on $[a, b]$. Again Lemma 2.3 yields

$$
\sum_{j, k=0}^{p-1} d_{j} d_{k}\left[(a+b) \omega_{j+k+1}-a b \omega_{j+k}-\omega_{j+k+2}\right]>0 .
$$

Therefore, the matrix $\left((a+b) \omega_{j+k+1}-a b \omega_{j+k}-\omega_{j+k+2}\right)_{j, k=0}^{p-1}$ is positive definite.

In the case $m$ odd we consider in the same way non-negative polynomials of the type $(t-a)\left(\sum_{i=0}^{l_{a}} c_{i} t^{i}\right)^{2}$ and $(b-t)\left(\sum_{i=0}^{l_{b}} d_{i} t^{i}\right)^{2}$.

\section{NeW DETERminantal INEQUALITIES FOR DUAL QUERMASSINTEGRALS}

Theorem 2.2 immediately leads to determinantal inequalities among the dual quermassintegrals $\widetilde{\mathrm{W}}_{i}(K, L), i \in \mathbb{N} \cup\{0\}$, of two star bodies $K, L \in \mathcal{S}_{0}^{n}$. To this end we just note that, in view of Remark 2.1. Theorem 2.2 gives that for every $m \in \mathbb{N}$ the following Hankel matrices are positive definite, except when $K$ and $L$ are dilates:

$$
\begin{aligned}
& \left(\widetilde{\mathrm{W}}_{j+k}(K, L)\right)_{0 \leq j, k \leq m},\left(\widetilde{\mathrm{W}}_{j+k+1}(K, L)-a \widetilde{\mathrm{W}}_{j+k}(K, L)\right)_{0 \leq j, k \leq m}, \\
& \left((a+b) \widetilde{\mathrm{W}}_{j+k+1}(K, L)-a b \widetilde{\mathrm{W}}_{j+k}(K, L)-\widetilde{\mathrm{W}}_{j+k+2}(K, L)\right)_{0 \leq j, k \leq m}, \\
& \left(b \widetilde{\mathrm{W}}_{j+k}(K, L)-\widetilde{\mathrm{W}}_{j+k+1}(K, L)\right)_{0 \leq j, k \leq m},
\end{aligned}
$$

where $a=\min _{u \in \mathbb{S}^{n-1}} \rho_{L}(u) / \rho_{K}(u), b=\max _{u \in \mathbb{S}^{n-1}} \rho_{L}(u) / \rho_{K}(u)$.

Theorem 1.2 establishes determinantal inequalities also for real indices.

Proof of Theorem 1.2. Obviously, if $K=\lambda L$ for some $\lambda>0$ then $\operatorname{det} A_{m}=$ 0 . Hence, we may assume that $K$ and $L$ are not dilates of each other. Let $f: \mathbb{S}^{n-1} \longrightarrow(0, \infty)$ be defined by

$$
f(u)=\frac{\rho_{L}(u)}{\rho_{K}(u)} .
$$

Since $K$ and $L$ are not dilates of each other, $f(u)$ is a continuous nonconstant function. 
Let $a_{i} \in \mathbb{R}, i=0, \ldots, m$, not all of them zero. Then it is known that the so-called generalized Dirichlet (or exponential) polynomial $\sum_{i=1}^{m} a_{i} x^{r_{i}}$ has only finitely many roots in $(0, \infty)$ (see, e.g., [15]). Hence

$$
P(x)=\left(\sum_{i=1}^{m} a_{i} x^{r_{i}}\right)^{2}=\sum_{i, j=1}^{m} a_{i} a_{j} x^{r_{i}+r_{j}}
$$

is non-negative for all $x \in(0, \infty)$ and has only finitely many zeros. Since $f(u)$ is a non-constant continuous function we conclude that

$$
\frac{1}{n} \int_{\mathbb{S}^{n-1}} P(f(u)) \rho_{K}^{n}(u) \mathrm{d} \sigma(u)>0 .
$$

Thus,

$$
\sum_{i, j=1}^{m} a_{i} a_{j}\left(\frac{1}{n} \int_{\mathbb{S}^{n-1}}\left(\frac{\rho_{L}(u)}{\rho_{K}(u)}\right)^{r_{i}+r_{j}} \rho_{K}^{n}(u) \mathrm{d} \sigma(u)\right)=\sum_{i, j=1}^{m} a_{i} a_{j} \widetilde{\mathrm{W}}_{r_{i}+r_{j}}(K, L)>0,
$$

which shows that $A_{m}$ is a positive definite matrix, and so $\operatorname{det} A_{m}>0$.

We observe that Theorem 1.2 implies, in particular, for $m=2$ and real $r \neq s \in \mathbb{R}$, that

$$
\begin{aligned}
0 & \leq \operatorname{det}\left(\begin{array}{cc}
\widetilde{\mathrm{W}}_{r}(K, L) & \widetilde{\mathrm{W}}_{(r+s) / 2}(K, L) \\
\widetilde{\mathrm{W}}_{(r+s) / 2}(K, L) & \widetilde{\mathrm{W}}_{s}(K, L)
\end{array}\right) \\
& =\widetilde{\mathrm{W}}_{r}(K, L) \widetilde{\mathrm{W}}_{s}(K, L)-\widetilde{\mathrm{W}}_{(r+s) / 2}(K, L)^{2}
\end{aligned}
$$

which might be considered as particular cases of the dual AleksandrovFenchel inequalities (2.1). Taking different submatrices we obtain different families of inequalities.

Remark 3.1. The determinantal inequalities in Corollary 1.1 cannot be obtained from the known Alexandrov-Fenchel inequalities (2.1). For instance, for any sequence of numbers $\omega_{0}>\omega_{1}>\omega_{2}=\omega_{3}=\omega_{4}$ (cf. Lemma 2.1 i)) satisfying (2.1), e.g., $(5,2,1,1,1)$, we have $\operatorname{det} \Delta_{2}<0$.

\section{The SET of Roots of DUAL Steiner Polynomials}

We start collecting some properties on the behavior of the roots of dual Steiner polynomials

$$
\widetilde{f}_{K, L}(z)=\sum_{i=0}^{n}\left(\begin{array}{l}
n \\
i
\end{array}\right) \widetilde{\mathrm{W}}_{i}(K, L) z^{i}, \quad z \in \mathbb{C}
$$

when the involved star bodies $K, L \in \mathcal{S}_{0}^{n}$ slightly change. They will be used for the proof of Theorem 1.3. We notice that these properties are similar to the ones of the relative Steiner polynomial, but for completeness' sake we include the proof.

Lemma 4.1. Let $K, L \in \mathcal{S}_{0}^{n}$, and let $\gamma$ be a root of $\widetilde{f}_{K, L}(z)$. 
i) Let $\lambda>0$. Then $\lambda \gamma$ is a root of $\widetilde{f}_{\lambda K, L}(z)$.

ii) Let $\mu \geq 0$. Then $\gamma-\mu$ is a root of $\widetilde{f}_{K \widetilde{+} \mu L, L}(z)$.

iii) Let $\gamma=\alpha+\beta$ i with $\alpha<0$, and let $0<\rho \leq 1$. Then $\alpha+(\rho \beta)$ i is a root of $\widetilde{f}_{\rho K \widetilde{+}(\rho-1) \alpha L, L}(z)$.

Proof. Since $\widetilde{\mathrm{W}}_{i}(\lambda K, L)=\lambda^{n-i} \widetilde{\mathrm{W}}_{i}(K, L)$ for any $i=0, \ldots, n$, we have $\widetilde{f}_{\lambda K, L}(z)=\lambda^{n} \widetilde{f}_{K, L}(z / \lambda)$, which shows i).

For $\lambda, \mu \geq 0$, the radial addition of star bodies satisfies $\mu L \widetilde{+} \lambda L=(\mu+\lambda) L$ (see e.g. [19, (2.2)]), and hence

$$
|(K \widetilde{+} \mu L) \widetilde{+} \lambda L|=|K \widetilde{+}(\mu+\lambda) L|=\sum_{i=0}^{n}\left(\begin{array}{c}
n \\
i
\end{array}\right) \widetilde{\mathrm{W}}_{i}(K, L)(\mu+\lambda)^{i} .
$$

Therefore, $\widetilde{f}_{K \widetilde{+} \mu L, L}(z)=\widetilde{f}_{K, L}(\mu+z)$, which implies ii).

Finally, iii) is just a combination of ii) and i), because the radial addition satisfies $\rho K \widetilde{+}(\rho-1) \alpha L=\rho(K \widetilde{+}[(\rho-1) \alpha / \rho] L)$ (see again [19, (2.2)]).

The following proposition states that both, the derivative as well as the antiderivative of a dual Steiner polynomial are again dual Steiner polynomials. This result will be crucial in the proof of Theorem 1.3 . We recall that for an integer $m \geq 1, C_{a, b}^{m}$ denotes the cone $C_{a, b}^{(0,1, \ldots, m)} \subset \mathbb{R}^{m+1}$.

Proposition 4.1. Let $K, L \in \mathcal{S}_{0}^{n}$.

i) There exist $K^{\prime}, L^{\prime} \in \mathcal{S}_{0}^{n-1}$ such that

$$
\frac{\mathrm{d} \widetilde{f}_{K, L}}{\mathrm{~d} z}(z)=\widetilde{f}_{K^{\prime}, L^{\prime}}(z) .
$$

ii) There exist $K^{\prime \prime}, L^{\prime \prime} \in \mathcal{S}_{0}^{n+1}$ such that

$$
\frac{\mathrm{d} \widetilde{f}_{K^{\prime \prime}, L^{\prime \prime}}}{\mathrm{d} z}(z)=\widetilde{f}_{K, L}(z)
$$

Proof. First, we note that since $\widetilde{\mathrm{W}}_{n-i}(K, L)=\widetilde{\mathrm{W}}_{i}(L, K)$ for all $i=0, \ldots, n$, Theorem 1.1 gives that

a) either there exist $0<a<b$ such that

$$
\left(\widetilde{\mathrm{W}}_{n}(K, L), \widetilde{\mathrm{W}}_{n-1}(K, L), \ldots, \widetilde{\mathrm{W}}_{0}(K, L)\right)^{\top} \in \operatorname{int} C_{a, b}^{n},
$$

b) or $\widetilde{\mathrm{W}}_{n-i}(K, L)=\lambda^{i} \widetilde{\mathrm{W}}_{n}(K, L)$ for some $\lambda>0$ and all $i=1, \ldots, n$.

For i) we observe that the derivative of $\widetilde{f}_{K, L}(z)$ is given by

$$
\frac{\mathrm{d} \widetilde{f}_{K, L}}{\mathrm{~d} z}(z)=\sum_{i=1}^{n}\left(\begin{array}{c}
n \\
i
\end{array}\right) i \widetilde{\mathrm{W}}_{i}(K, L) z^{i-1}=\sum_{i=0}^{n-1}\left(\begin{array}{c}
n-1 \\
i
\end{array}\right) n \widetilde{\mathrm{W}}_{i+1}(K, L) z^{i} .
$$

If a) holds then, in particular,

$$
\left(\widetilde{\mathrm{W}}_{n}(K, L), \widetilde{\mathrm{W}}_{n-1}(K, L), \ldots, \widetilde{\mathrm{W}}_{1}(K, L)\right)^{\top} \in \operatorname{int} C_{a, b}^{n-1},
$$


and thus $\left(n \widetilde{\mathrm{W}}_{n}(K, L), n \widetilde{\mathrm{W}}_{n-1}(K, L), \ldots, n \widetilde{\mathrm{W}}_{1}(K, L)\right)^{\top} \in \operatorname{int} C_{a, b}^{n-1}$. Hence, by Theorem 1.1, there exist star bodies $K^{\prime}, L^{\prime} \in \mathcal{S}_{0}^{n-1}$ such that

$$
n \widetilde{\mathrm{W}}_{n-i}(K, L)=\widetilde{\mathrm{W}}_{i}^{(n-1)}\left(L^{\prime}, K^{\prime}\right)=\widetilde{\mathrm{W}}_{n-i-1}^{(n-1)}\left(K^{\prime}, L^{\prime}\right)
$$

for all $i=0, \ldots, n-1$, where $\widetilde{\mathrm{W}}_{i}^{(j)}$ denotes the $i$-th dual quermassintegral in $\mathbb{R}^{j}$. Therefore $\left(\mathrm{d} \widetilde{f}_{K, L} / \mathrm{d} z\right)(z)$ is the dual Steiner polynomial in $\mathbb{R}^{n-1}$ of the bodies $K^{\prime}, L^{\prime}$.

If b) holds, then $K=\lambda L$ (cf. Lemma 2.1 ii)), and hence

$$
\frac{\mathrm{d} \widetilde{f}_{K, L}}{\mathrm{~d} z}(z)=n|L|(\lambda+z)^{n-1}
$$

Taking $L^{\prime} \in \mathcal{S}_{0}^{n-1}$ with $\left|L^{\prime}\right|_{n-1}=n|L|$ and setting $K^{\prime}=\lambda L^{\prime}$, we obtain

$$
\widetilde{f}_{K^{\prime}, L^{\prime}}(z)=\sum_{i=0}^{n-1}\left(\begin{array}{c}
n-1 \\
i
\end{array}\right) \widetilde{\mathrm{W}}_{i}^{(n-1)}\left(K^{\prime}, L^{\prime}\right) z^{i}=\left|L^{\prime}\right|_{n-1}(\lambda+z)^{n-1}=\frac{\mathrm{d} \widetilde{f}_{K, L}}{\mathrm{~d} z}(z) .
$$

This finishes the proof of i).

The formal antiderivative of $\widetilde{f}_{K, L}(z)$ is given by

$$
\begin{aligned}
\int \widetilde{f}_{K, L}(z) \mathrm{d} z & =C+\sum_{i=0}^{n}\left(\begin{array}{c}
n \\
i
\end{array}\right) \frac{1}{i+1} \widetilde{\mathrm{W}}_{i}(K, L) z^{i+1} \\
& =C+\sum_{i=1}^{n+1}\left(\begin{array}{c}
n+1 \\
i
\end{array}\right) \frac{1}{n+1} \widetilde{\mathrm{W}}_{i-1}(K, L) z^{i}
\end{aligned}
$$

for a constant $C \in \mathbb{R}$.

Again we start assuming that a) holds. Let $H=\operatorname{span}\left\{\mathrm{e}_{1}, \ldots, \mathrm{e}_{n+1}\right\} \subset$ $\mathbb{R}^{n+2}$ be the linear subspace spanned by the first $n+1$ canonical vectors $\mathrm{e}_{i}$. Since

$$
\frac{1}{n+1}\left(\widetilde{\mathrm{W}}_{n}(K, L), \widetilde{\mathrm{W}}_{n-1}(K, L), \ldots, \widetilde{\mathrm{W}}_{0}(K, L)\right)^{\top} \in \operatorname{int} C_{a, b}^{n}
$$

and $C_{a, b}^{n}=C_{a, b}^{n+1} \mid H$ is the orthogonal projection of $C_{a, b}^{n+1}$ onto $H$, there exists a point $\left(\omega_{0}, \ldots, \omega_{n+1}\right)^{\top} \in \operatorname{int} C_{a, b}^{n+1}$ such that

$$
\left(\omega_{0}, \ldots, \omega_{n+1}\right)^{\top} \mid H=\frac{1}{n+1}\left(\widetilde{\mathrm{W}}_{n}(K, L), \widetilde{\mathrm{W}}_{n-1}(K, L), \ldots, \widetilde{\mathrm{W}}_{0}(K, L)\right)^{\top} .
$$

By Theorem 1.1. there exist star bodies $L^{\prime \prime}, K^{\prime \prime} \in \mathcal{S}_{0}^{n+1}$ such that $\omega_{i}=$ $\widetilde{\mathrm{W}}_{i}^{(n+1)}\left(L^{\prime \prime}, K^{\prime \prime}\right), i=0, \ldots, n+1$, and, consequently,

$$
\frac{1}{n+1} \widetilde{\mathrm{W}}_{n-i}(K, L)=\omega_{i}=\widetilde{\mathrm{W}}_{i}^{(n+1)}\left(L^{\prime \prime}, K^{\prime \prime}\right)=\widetilde{\mathrm{W}}_{n+1-i}^{(n+1)}\left(K^{\prime \prime}, L^{\prime \prime}\right),
$$


for all $i=0, \ldots, n$. Then, setting $C=\omega_{n+1}$ we get

$$
\begin{aligned}
\widetilde{f}_{K^{\prime \prime}, L^{\prime \prime}}(z) & =\sum_{i=0}^{n+1}\left(\begin{array}{c}
n+1 \\
i
\end{array}\right) \widetilde{\mathrm{W}}_{i}^{(n+1)}\left(K^{\prime \prime}, L^{\prime \prime}\right) z^{i} \\
& =C+\sum_{i=1}^{n+1}\left(\begin{array}{c}
n+1 \\
i
\end{array}\right) \frac{1}{n+1} \widetilde{\mathrm{W}}_{i-1}(K, L) z^{i},
\end{aligned}
$$

and thus $\left(\mathrm{d} \widetilde{f}_{K^{\prime \prime}, L^{\prime \prime}} / \mathrm{d} z\right)(z)=\widetilde{f}_{K, L}(z)$.

Finally, if b) holds, then $K=\lambda L$ (cf. Lemma 2.1 ii)). So it suffices to take $L^{\prime \prime} \in \mathcal{S}_{0}^{n+1}$ with $\left|L^{\prime \prime}\right|_{n+1}=|L| /(n+1)$ and to set $K^{\prime \prime}=\lambda L^{\prime \prime}$. Then

$$
\begin{aligned}
\frac{\mathrm{d} \widetilde{f}_{K^{\prime \prime}, L^{\prime \prime}}}{\mathrm{d} z}(z) & =\frac{\mathrm{d}}{\mathrm{d} z}\left[\sum_{i=0}^{n+1}\left(\begin{array}{c}
n+1 \\
i
\end{array}\right) \widetilde{\mathrm{W}}_{i}^{(n+1)}\left(K^{\prime \prime}, L^{\prime \prime}\right) z^{i}\right] \\
& =\frac{\mathrm{d}}{\mathrm{d} z}\left[\left|L^{\prime \prime}\right|_{n+1}(\lambda+z)^{n+1}\right]=(n+1)\left|L^{\prime \prime}\right|_{n+1}(\lambda+z)^{n} \\
& =|L|(\lambda+z)^{n}=\widetilde{f}_{K, L}(z) .
\end{aligned}
$$

For the proof of Theorem 1.3 we need one more notation: for complex numbers $z_{1}, \ldots, z_{k} \in \mathbb{C}$ let

$$
\sigma_{i}\left(z_{1}, \ldots, z_{k}\right)=\sum_{\substack{J \subseteq\{1, \ldots, k\} \\ \# J=i}} \prod_{j \in J} z_{j}
$$

be the $i$-th elementary symmetric function of $z_{1}, \ldots, z_{k}, 1 \leq i \leq k$, and we set $\sigma_{0}\left(z_{1}, \ldots, z_{k}\right)=1$.

Proof of Theorem 1.3. For a) we firstly observe that -1 is a root of $\widetilde{f}_{L, L}(z)=$ $|L|(z+1)^{n}, L \in \mathcal{S}_{0}^{n}$, and thus, by Lemma $\left.4.1 \mathrm{i}\right)$, every $-c \in \mathbb{R}_{<0}, c>0$, will be a root of $\widetilde{f}_{c L, L}(z)$. Hence, $\widetilde{\mathcal{R}}(n)$ contains the negative real axis $\mathbb{R}_{<0}$.

By Lemma 4.1 i) we also know that $\lambda \gamma \in \widetilde{\mathcal{R}}(n)$ for all $\gamma \in \widetilde{\mathcal{R}}(n)$ and $\lambda>0$. It remains to show that $\widetilde{\mathcal{R}}(n)$ is convex. To this end, let $\gamma_{i}=\alpha_{i}+\beta_{i} \mathrm{i} \in \widetilde{\mathcal{R}}(n)$, $i=1,2$, and let $\rho \in(0,1)$.

If both roots are real, then $\gamma_{1}, \gamma_{2} \in \mathbb{R}_{<0}$ and we are done since $\widetilde{\mathcal{R}}(n)$ contains $\mathbb{R}_{<0}$. So we may assume that $\beta_{2} \geq \beta_{1} \geq 0, \beta_{2}>0$, and first we argue that there exist $K_{i}, L \in \mathcal{S}_{0}^{n}, i=1,2$, such that $\gamma_{i}$ is a root of $\widetilde{f}_{K_{i}, L}(z)$, $i=1,2$.

If $\beta_{1}=0$ then $\alpha_{1}<0$ and we can set $K_{1}=\left|\alpha_{1}\right| L$ to get $\widetilde{f}_{K_{1}, L}\left(\gamma_{1}\right)=0$. So we suppose $\beta_{1}>0$.

First we assume $\left(\beta_{1} / \beta_{2}\right) \alpha_{2}-\alpha_{1} \geq 0$. Let $K_{2}, L \in \mathcal{S}_{0}^{n}$ be star bodies such that $\widetilde{f}_{K_{2}, L}\left(\gamma_{2}\right)=0$. With $\lambda=\beta_{1} / \beta_{2}$ and Lemma $\left.4.1 \mathrm{i}\right)$, we find that $\lambda \gamma_{2}=\lambda \alpha_{2}+\beta_{1}$ i is a root of $\tilde{f}_{\lambda K_{2}, L}(z)$ (see Figure 1).

By assumption, $\mu=\lambda \alpha_{2}-\alpha_{1} \geq 0$, and Lemma 4.1 ii) ensures that $\gamma_{1}=\lambda \gamma_{2}-\mu$ is a root of $\widetilde{f}_{\lambda K_{2} \tilde{+} \mu L, L}(z)$. Thus, with $K_{1}=\lambda K_{2} \widetilde{+} \mu L$ we get 


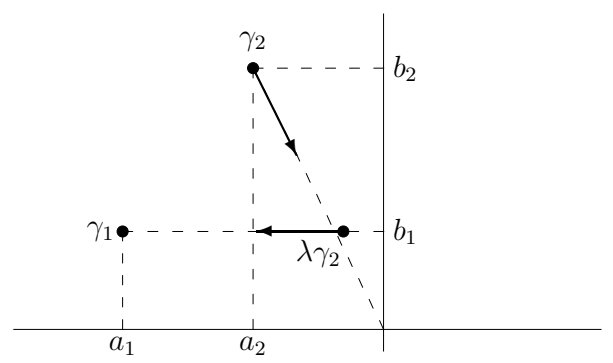

Figure 1. Constructing $K_{1} \in \mathcal{S}_{0}^{n}$ such that $\tilde{f}_{K_{1}, L}\left(\gamma_{1}\right)=0$.

$\widetilde{f}_{K_{1}, L}\left(\gamma_{1}\right)=0$. If $\left(\beta_{1} / \beta_{2}\right) \alpha_{2}-\alpha_{1}<0$ then $\left(\beta_{2} / \beta_{1}\right) \alpha_{1}-\alpha_{2}>0$ and we interchange the roles of $K_{1}$ and $K_{2}$.

Finally, in order to prove the convexity of the cone, we just have to construct a star body $M \in \mathcal{S}_{0}^{n}$ such that $\rho \gamma_{1}+(1-\rho) \gamma_{2}$ is a root of $\widetilde{f}_{M, L}(z)$. Without loss of generality let $\alpha_{1} / \beta_{1}=\max \left\{\alpha_{i} / \beta_{i}: \beta_{i}>0, i=1,2\right\}$ and let $\mu=\beta_{1} /\left(\rho \beta_{1}+(1-\rho) \beta_{2}\right)$. Then

$$
\nu=\mu\left(\rho \alpha_{1}+(1-\rho) \alpha_{2}\right)=\beta_{1} \frac{\rho \alpha_{1}+(1-\rho) \alpha_{2}}{\rho \beta_{1}+(1-\rho) \beta_{2}} \leq \alpha_{1},
$$

and Lemma 4.1 ii) ensures that $\nu+\beta_{1}$ i is a root of $\widetilde{f}_{K_{1}+\left(\alpha_{1}-\nu\right) L, L}(z)$. Finally, Lemma 4.1 i) shows that $\rho \gamma_{1}+(1-\rho) \gamma_{2}$ is a root of the dual Steiner polynomial $\widetilde{f}_{M, L}(z)$ for $M=(1 / \mu)\left(K_{1} \widetilde{+}\left(\alpha_{1}-\nu\right) L\right)$.

Next in order to prove b), i.e., to prove that $\widetilde{\mathcal{R}}(n)$ is half-open, we are going to show that $C=\mathbb{C}^{+} \backslash \widetilde{\mathcal{R}}(n)$ is closed. Let $\left(z_{i}\right)_{i \in \mathbb{N}} \subset C$ be a convergent sequence with $z=\lim _{i \rightarrow \infty} z_{i}$ and we have to prove that $z \notin \widetilde{\mathcal{R}}(n)$.

For the sake of brevity we denote, for $n \geq 3$, by $\Gamma$ any $(n-2)$-tuple of complex numbers of the form

$$
\Gamma= \begin{cases}\left(\gamma_{2}, \bar{\gamma}_{2}, \ldots, \gamma_{n / 2}, \bar{\gamma}_{n / 2}\right)^{\top} \in \mathbb{C}^{n-2}, & \text { if } n \text { is even, } \\ \left(\gamma_{2}, \bar{\gamma}_{2}, \ldots, \gamma_{(n-1) / 2}, \bar{\gamma}_{(n-1) / 2}, c\right)^{\top} \in \mathbb{C}^{n-3} \times \mathbb{R}_{<0}, & \text { if } n \text { is odd. }\end{cases}
$$

Let $\sigma: \mathbb{C}^{n} \longrightarrow \mathbb{C}^{n} \times\{1\}$ be the continuous map given by

$$
\sigma=\left((-1)^{n} \sigma_{n},(-1)^{n-1} \frac{\sigma_{n-1}}{n}, \ldots,(-1)^{i} \frac{\sigma_{i}}{\left(\begin{array}{c}
n \\
i
\end{array}\right)}, \ldots,-\frac{\sigma_{1}}{n}, 1\right)^{\top} .
$$

For an arbitrary but fixed $(n-2)$-tuple $\Gamma$, let $\sigma\left(z_{i}, \bar{z}_{i}, \Gamma\right)=\left(\omega_{0}^{i}, \ldots, \omega_{n-1}^{i}, 1\right)^{\top}$ for all $i \in \mathbb{N}$. Since $z_{i}$ is not a root of any dual Steiner polynomial, $\omega_{j}^{i}$, $j=0, \ldots, n$, with $\omega_{n}^{i}=1$, cannot be dual quermassintegrals of any pair of star bodies. Hence, Theorem 1.1 implies that $\left(\omega_{0}^{i}, \ldots, \omega_{n-1}^{i}, 1\right)^{\top} \notin \operatorname{int} C_{a, b}^{n}$ for any $0<a<b$, and that there exists no $\lambda>0$ such that $\omega_{j}^{i}=\lambda^{j} \omega_{0}^{i}$. 
On the other hand, since $\sigma$ is continuous, $\left(\omega_{0}^{i}, \ldots, \omega_{n-1}^{i}, 1\right)_{i \in \mathbb{N}}^{\top}$ is a convergent sequence and

$$
\sigma(z, \bar{z}, \Gamma)=\lim _{i \rightarrow \infty}\left(\omega_{0}^{i}, \ldots, \omega_{n-1}^{i}, 1\right)^{\top}=:\left(\omega_{0}, \ldots, \omega_{n-1}, 1\right)^{\top} \notin \operatorname{int} C_{a, b}^{n}
$$

for any $0<a<b$. Moreover, if there exists $\lambda>0$ such that $\omega_{j}=\lambda^{j} \omega_{0}$, $j=0, \ldots, n$, then $\omega_{j}=\widetilde{\mathrm{W}}_{j}(\lambda K, K)$ for some $K \in \mathcal{S}_{0}^{n}$, and so $z$ would be a root of the dual Steiner polynomial $\tilde{f}_{\lambda K, K}(z)$. Hence, $z \in \mathbb{R}_{<0}$, which leads to the contradiction $z_{i} \in \widetilde{\mathcal{R}}(n)$ for large $i$.

Since this holds for any $(n-2)$-tuple $\Gamma$, we can conclude that $z \notin \widetilde{\mathcal{R}}(n)$.

Finally, we show c), i.e., $\widetilde{\mathcal{R}}(n) \subseteq \widetilde{\mathcal{R}}(n+1)$. To this end, let $\gamma \in \widetilde{\mathcal{R}}(n)$. Then there exists a dual Steiner polynomial $\widetilde{f}_{K, L}(z)$ for $K, L \in \mathcal{S}_{0}^{n}$, such that $\widetilde{f}_{K, L}(\gamma)=0$. By Proposition 4.1 ii), we know there are star bodies $K^{\prime \prime}, L^{\prime \prime} \in \mathcal{S}_{0}^{n+1}$ satisfying

$$
\frac{\mathrm{d} \widetilde{f}_{K^{\prime \prime}, L^{\prime \prime}}}{\mathrm{d} z}(z)=\widetilde{f}_{K, L}(z)
$$

Denoting by $\gamma_{1}, \ldots, \gamma_{n+1}$ the roots of $\widetilde{f}_{K^{\prime \prime}, L^{\prime \prime}}(z)$, Lucas' theorem (see e.g. [22, Theorem $(6,1)]$ ) on the location of the roots of the derivative of a polynomial implies that

$$
\gamma \in \operatorname{conv}\left\{\gamma_{1}, \ldots, \gamma_{n+1}\right\}
$$

Since $\widetilde{\mathcal{R}}(n+1)$ is convex, we get $\gamma \in \widetilde{\mathcal{R}}(n+1)$.

In general, it seems to be hard to describe explicitly the cones $\widetilde{\mathcal{R}}(n)$. In dimension 2 we have the following representation.

Proposition 4.2. $\widetilde{\mathcal{R}}(2)=\left\{z \in \mathbb{C}^{+}: \operatorname{Re}(z)<0\right\}$.

Proof. Obviously, since $\widetilde{f}_{K, L}(z)=\widetilde{\mathrm{W}}_{0}(K, L)+2 \widetilde{\mathrm{W}}_{1}(K, L) z+\widetilde{\mathrm{W}}_{2}(K, L) z^{2}$, $K, L \in \mathcal{S}_{0}^{2}$, has only positive coefficients, the roots have negative real parts. Moreover, according to the Aleksandrov-Fenchel inequality (2.1), the discriminant $\widetilde{\mathrm{W}}_{1}(K, L)^{2}-\widetilde{\mathrm{W}}_{0}(K, L) \widetilde{\mathrm{W}}_{2}(K, L)$ vanishes, i.e., the roots are real, if and only if $K$ and $L$ are dilates.

Now let $\gamma=\alpha+\beta \mathrm{i} \in\left\{z \in \mathbb{C}^{+}: \operatorname{Re}(z)<0\right\}$. If $\beta=0$, then $\gamma$ is a root of $\widetilde{f}_{|\alpha| K, K}(z)$ for $K \in \mathcal{S}_{0}^{2}$. So let $\beta>0$ and we set

$$
\omega_{0}=\alpha^{2}+\beta^{2}, \quad \omega_{1}=-\alpha \quad \text { and } \quad \omega_{2}=1 .
$$

Then $\gamma$ is a root of $\omega_{2} z^{2}+2 \omega_{1} z+\omega_{0}$, and this polynomial is a dual Steiner polynomial of two planar star bodies if there exist $0<a<b$ with (cf. Theorem 1.1)

$$
\left(\omega_{0}, \omega_{1}, \omega_{2}\right)^{\top} \in \operatorname{int} C_{a, b}^{3}=\operatorname{int} \operatorname{pos}\left\{\left(1, t, t^{2}\right)^{\top}: t \in[a, b]\right\} .
$$

Hence, $\gamma$ is root of a dual Steiner-polynomial if

$$
\left(\frac{\omega_{1}}{\omega_{0}}, \frac{\omega_{2}}{\omega_{0}}\right)^{\top} \in \operatorname{int} \operatorname{conv}\left\{\left(t, t^{2}\right)^{\top}: t>0\right\}
$$


(cf. Remark 2.2), which is equivalent to $\omega_{2} / \omega_{0}>\left(\omega_{1} / \omega_{0}\right)^{2}$ or $\omega_{1}^{2}<\omega_{2} \omega_{0}$. Since $\beta>0$ this is true, and so $\gamma$ is a root of a dual Steiner polynomial.

The different behavior of the roots of dual Steiner polynomials compared to the ones of (classical) Steiner polynomials shows up also in the stability of the polynomials. We recall that a real polynomial whose zeros all have negative real parts is called stable or Hurwitz. In [13, Proposition 1.3] it was shown that the Steiner polynomial is stable if and only if $n \leq 9$.

Proposition 4.3. A dual Steiner polynomial is stable if and only if $n=2$.

Proof. Proposition 4.2 shows that dual Steiner polynomials are stable for $n=2$. In order to show that for $n \geq 3$ there exist dual Steiner polynomials having roots with positive real parts, it suffices to consider $n=3$ (cf. Theorem $1.3 \mathrm{c})$ ). Let $\alpha(t)=\left(t, t^{2}, t^{3}\right)^{\top} \subset \mathbb{R}^{3}$ be the moment curve. Since any four points on this moment curve are affinely independent, we have

$$
\frac{1}{4}(\alpha(1)+\alpha(2)+\alpha(3)+\alpha(4))+\in \operatorname{int} \operatorname{conv}\left\{\left(t, t^{2}, t^{3}\right)^{\top}: t \in[1,4]\right\} .
$$

The coordinates of the point on the left hand side are $(5 / 2,15 / 2,25)^{\top}$ and with Theorem 1.1 we see that the polynomial

$$
f(z)=1+\frac{5}{2} z+\frac{15}{2} z^{2}+25 z^{3}
$$

is a dual Steiner polynomial. Numerical computations show that the complex roots have positive real parts.

As shown in [13, Proposition 2.3], a Steiner polynomial of degree $n$ can have $n$ distinct real roots. In the dual setting this cannot occur.

Proposition 4.4. Let $K, L \in \mathcal{S}_{0}^{n}$. All roots of $\widetilde{f}_{K, L}(z)$ are real if and only if $K=\lambda L$ for some $\lambda>0$, i.e., all roots are equal.

Proof. Let $\gamma_{1}, \ldots, \gamma_{n} \in \mathbb{R}_{<0}$ be the roots of $\widetilde{f}_{K, L}(z)=0$. Then the elementary symmetric means of the roots satisfy the Newton inequalities (see, e.g., [11]), i.e.,

$$
\left(\frac{\sigma_{j}\left(\gamma_{1}, \ldots, \gamma_{n}\right)}{\left(\begin{array}{c}
n \\
j
\end{array}\right)}\right)^{2} \geq \frac{\sigma_{j-1}\left(\gamma_{1}, \ldots, \gamma_{n}\right)}{\left(\begin{array}{c}
n \\
j-1
\end{array}\right)} \frac{\sigma_{j+1}\left(\gamma_{1}, \ldots, \gamma_{n}\right)}{\left(\begin{array}{c}
n \\
j+1
\end{array}\right)}
$$

Since

$$
\sigma_{j}\left(\gamma_{1}, \ldots, \gamma_{n}\right)=(-1)^{j}\left(\begin{array}{c}
n \\
j
\end{array}\right) \frac{\widetilde{\mathrm{W}}_{n-j}(K, L)}{\widetilde{\mathrm{W}}_{n}(K, L)},
$$

the above inequality translates into

$$
\widetilde{\mathrm{W}}_{n-j}(K, L)^{2} \geq \widetilde{\mathrm{W}}_{n-j+1}(K, L) \widetilde{\mathrm{W}}_{n-j-1}(K, L) .
$$

In view of the dual Aleksandrov-Fenchel inequalities (2.1) we must have the equality $\widetilde{\mathrm{W}}_{n-j}(K, L)^{2}=\widetilde{\mathrm{W}}_{n-j+1}(K, L) \widetilde{\mathrm{W}}_{n-j-1}(K, L)$, and thus $K=\lambda L$ for some $\lambda>0$. 


\section{REFERENCES}

[1] A. Bernig, The isoperimetrix in the dual Brunn-Minkowski theory, Adv. Math. 254 (2014), 1-14.

[2] K. J. Böröczky, M. Henk, H. Pollehn, Subspace concentration of dual curvature measures, to appear in J. Differential Geometry.

[3] Yu. D. Burago, V. A. Zalgaller, Geometric Inequalities. Springer, Berlin, 1988 (Russian original: 1980).

[4] P. Dulio, R. J. Gardner, C. Peri, Characterizing the dual mixed volume via additive functionals, Indiana Univ. Math. J. 65 (1) (2016), 69-91.

[5] W. J. Firey, p-means of convex bodies, Math. Scand. 10 (1962), 17-24.

[6] R. J. Gardner, A positive answer to the Busemann-Petty problem in three dimensions, Ann. Math. 140 (2) (1994), 435-447.

[7] R. J. Gardner, The dual Brunn-Minkowski theory for bounded Borel sets: dual affine quermassintegrals and inequalities, Adv. Math. 216 (1) (2007), 358-386.

[8] R. J. Gardner, D. Hug, W. Weil, Operations between sets in geometry, J. Eur. Math. Soc. 15 (6) (2013), 2297-2352.

[9] R. J. Gardner, D. Hug, W. Weil, The Orlicz-Brunn-Minkowski theory: a general framework, additions, and inequalities, J. Differ. Geom. 97 (3) (2014), 427-476.

[10] R. J. Gardner, A. Koldobsky, T. Schlumprecht, An analytic solution to the BusemannPetty problem on sections of convex bodies, Ann. Math. 149 (2) (1999), 691-703.

[11] G. Hardy, J. E. Littlewood, G. Pólya, Inequalities. Reprint of the 1952 edition. Cambridge Mathematical Library, Cambridge University Press, Cambridge, 1988.

[12] R. Heine, Der Wertvorrat der gemischten Inhalte von zwei, drei und vier ebenen Eibereichen, Math. Ann. 115 (1937), 115-129.

[13] M. Henk, M. A. Hernández Cifre, E. Saorín, Steiner polynomials via ultra-logconcave sequences, Commun. Contemp. Math. 14 (6) (2012), 1-16.

[14] Y. Huang, E. Lutwak, D. Yang, G. Zhang, Geometric measures in the dual BrunnMinkowski theory and their associated Minkowski problems, Acta Math. 216 (2) (2016), 325-388.

[15] G. J. O. Jameson, Counting zeros of generalized polynomials: Descartes' rule of signs and Laguerre's extensions, Math. Gazette 90 (518) (2006), 223-234.

[16] D. A. Klain, Star valuations and dual mixed volumes, Adv. Math. 121 (1) (1996), 80-101.

[17] M. G. Krein, A. A. Nudelman, The Markov moment problem and extremal problems. Ideas and problems of P. L. Chebyshev and A. A. Markov and their further development. Translations of Mathematical Monographs, vol. 50, American Mathematical Society, Providence, R.I., 1977.

[18] E. Lutwak, Dual mixed volumes, Pacific J. Math. 58 (2) (1975), 531-538.

[19] E. Lutwak, Dual cross-sectional measures, Atti Accad. Naz. Lincei 58 (1975), 1-5.

[20] E. Lutwak, The Brunn-Minkowski-Firey theory, I, J. Differential Geom. 38 (1) (1993), 131-150.

[21] E. Lutwak, The Brunn-Minkowski-Firey theory, II, Adv. Math. 118 (2) (1996), 244294.

[22] M. Marden, Geometry of polynomials. 2nd edition, Mathematical Surveys, no. 3, American Mathematical Society, Providence, R.I., 1966.

[23] R. Puente, Cyclic convex bodies and optimization moment problems, Linear Algebra Appl. 426 (2-3) (2007), 596-609.

[24] K. Schmüdgen, The Moment Problem. Graduate Texts in Mathematics 277, Springer, Cham, 2017

[25] R. Schneider, Convex bodies: The Brunn-Minkowski theory. 2nd expanded edition, Cambridge University Press, Cambridge, 2014. 
[26] G. C. Shephard, Inequalities between mixed volumes of convex sets, Mathematika 7 (1960), 125-138.

[27] J. Steiner, Über parallele Flächen, Monatsber. Preuss. Akad. Wiss. (1840), 114-118, [Ges. Werke, Vol II (Reimer, Berlin, 1882) 245-308].

[28] G. Zhang, A positive solution to the Busemann-Petty problem in $\mathbb{R}^{4}$, Ann. Math. 149 (2) (1999), 535-543.

Departamento de Matemáticas, Universidad de Zaragoza, C/ Pedro CerBUNA 12, 50009-ZARAgOZA, SPAIN

E-mail address: alonsod@unizar.es

Institut für Mathematik, Technische Universität Berlin, Strasse des 17. Juni 136, D-10623 Berlin, Germany

E-mail address: henk@math.tu-berlin.de

Departamento de Matemáticas, Universidad de Murcia, Campus de EspinarDO, 30100-Murcia, Spain

E-mail address: mhcifre@um.es 